\title{
SPAG9 is involved in hepatocarcinoma cell migration and invasion via modulation of ELKI expression
}

\author{
This article was published in the following Dove Press journal: \\ OncoTargets and Therapy \\ I March 2016 \\ Number of times this article has been viewed
}

\section{Qiuyue Yan ${ }^{1,2}$ \\ Guohua Lou ${ }^{3}$ \\ Ying Qian' \\ Bo Qin' \\ Xiuping $X u^{1,2}$ \\ Yanan Wang ${ }^{1,2}$ \\ Yanning Liu $^{3}$ \\ Xuejun Dong'}

'Shaoxing People's Hospital, Shaoxing Hospital Zhejiang University, Shaoxing, Zhejiang, ${ }^{2}$ The Key Laboratory of Laboratory Medicine, Ministry of Education of China, School of Laboratory Medicine and Life Science, Wenzhou Medical University, Wenzhou, ${ }^{3}$ State Key Laboratory for Diagnosis and Treatment of Infectious Diseases, Collaborative Innovation Center for Diagnosis and Treatment of Infectious Diseases, The First Affiliated Hospital, School of Medicine, Zhejiang University, Hangzhou, Zhejiang, People's Republic of China

Correspondence: Xuejun Dong Shaoxing People's Hospital, Shaoxing Hospital Zhejiang University, 568 Zhongxing North Road, Shaoxing, Zhejiang 312000, People's Republic of China

Tel/fax +86 57588228576

Email dxj9666@।63.com

Yanning Liu

State Key Laboratory for Diagnosis and Treatment of Infectious Diseases, Collaborative Innovation Center for Diagnosis and Treatment of Infectious Diseases, The First Affiliated Hospital, Zhejiang University, 79 Qingchun Road, 6A-17, Hangzhou, Zhejiang 310000,

People's Republic of China

Tel/fax +86 57I 87236579

Email rainyliu2002@163.com
Background: Sperm-associated antigen 9 (SPAG9) is upregulated in several malignancies and its overexpression is positively correlated with cancer cell malignancies. However, the specific biological roles of SPAG9 in hepatocellular carcinoma (HCC) are less understood.

Methods: We analyzed SPAG9 and ETS-like gene 1, tyrosine kinase (ELK1) expression in 50 paired $\mathrm{HCC}$ specimens and adjacent noncancerous liver specimens using immunohistochemistry. SPAG9 small interfering RNA (siRNA) was used to knockdown SPAG9 expression in HCCLM3 and HuH7 cell lines. We used plasmids to upregulate ELK1 expression and siRNA to downregulate $E L K 1$ expression in $\mathrm{HuH7}$ cells. Quantitative real-time polymerase chain reaction and Western blot were used to evaluate the expression of SPAG9 and ELK1 at the mRNA and protein level, respectively. Wound healing, matrigel migration, and invasion analyses were performed to determine the effect of SPAG9 and ELK1 on HCC metastasis.

Results: SPAG9 and ELK1 were overexpressed in HCC tissue specimens and their expressions were higher in HCCLM3 and HuH7 cells compared to the low-metastatic HepG2 cells. Overexpression of SPAG9 was positively associated with tumor-node-metastasis staging $(P=0.032)$, metastasis parameters $(P=0.018)$ of HCC patients, and ELK1 expression $(r=0.422, P<0.001)$ in HCC tissue specimens. In addition, knockdown of SPAG9 in HCCLM3 and HuH7 cells using siRNA significantly suppressed cell migration and invasion. Furthermore, we observed inhibition of ELK1 expression and p38 signaling. However, ELK1 overexpression reversed the inhibitory effects of SPAG9 siRNA on HCC cell metastasis and ELK1 depletion inhibited $\mathrm{HuH7}$ cell migration and invasion.

Conclusion: SPAG9 overexpression was positively correlated with HCC metastasis and SPAG9-induced migration and invasion were partially dependent on ELK1 expression in HCC cell lines. These results suggest that $S P A G 9$ may be a potential anti-metastasis target effective in HCC therapy.

Keywords: SPAG9, ELK1, hepatocellular carcinoma, metastasis

\section{Introduction}

Hepatocellular carcinoma (HCC) is the fifth most prevalent cancer and the third most common cause of cancer-related deaths worldwide. ${ }^{1}$ There are several treatment options for HCC patients, including surgical resection, radiotherapy, chemoembolization, and liver transplantation. Despite the numerous treatments available, HCC prognosis remains poor because of the high incidence of recurrence and metastasis. ${ }^{2}$ Therefore, more complete understanding of the mechanism involved in metastasis and the subsequent identification of novel therapeutic targets are necessary.

Cancer-testis (CT) antigens are a unique family of tumor antigens that have aberrant expression in various tumors and restricted expression in normal testis. ${ }^{3} \mathrm{CT}$ antigens have been reported to play an important role in a number of malignant properties of cancer cells. ${ }^{4,5}$ Sperm-associated antigen 9 (SPAG9) protein, a new member of CT antigen family, 
is highly expressed in $88 \%$ of breast cancer, $82 \%$ of cervical cancer, $74 \%$ of colorectal cancer, and $60 \%$ of astrocytoma. ${ }^{6-9}$ Its high expression is associated with lymph node metastasis in breast, prostate, and lung carcinomas, and SPAG9 small interfering RNA (siRNA) treatment could reduce the cellular growth, migration, and invasion in these cancers. ${ }^{10-12}$ Previous studies have shown that SPAG9 is involved in c-Jun-NH2kinase-signaling module and functions as a scaffolding protein for c-Jun-NH2-kinase binding and likely plays an important regulatory role in cell survival, proliferation, and tumor development. ${ }^{11,13,14}$ Recently, SPAG9 was identified as a novel binding partner required for protein kinase $\mathrm{C}$-related kinase 1 (PRK1)-mediated p38/ETS-like gene 1, tyrosine kinase (ELK1) activation in prostate cancer. ${ }^{15}$ Furthermore, p38 mitogen-activated protein kinase (MAPK) signaling activated transcription factors, including the ETS domain-containing protein ELK1, ${ }^{16}$, ${ }^{17}$ a modulator of tumor metastasis. ${ }^{18-21}$ However, the mechanism in which SPAG9 promotes HCC metastasis by affecting cell migration and invasion remains unclear.

In this study, we showed that SPAG9 silenced by siRNA suppressed HCC migration and invasion. In addition, our results suggest that these effects may have resulted from the modulation of ELK1 and phosphorylated p38 expression.

\section{Methods}

\section{Patients and specimens}

Specimens of HCC tissues were obtained from 50 patients who underwent hepatic surgical resection without preoperative systemic chemotherapy at Shaoxing People's Hospital (Shaoxing, People's Republic of China). These specimens were collected from the biological specimen bank in Shaoxing People's Hospital after written informed consent was obtained from the patients. The Ethics Committee of Shaoxing People's Hospital approved this study. The main clinical and pathological information are as follows: 39 males and eleven females, aged 35-79 (50.5 49.3$)$ years, 36 cases of hepatitis B surface antigen ( $\mathrm{HBsAg}$ )-positive patients, 30 cases of liver cirrhosis, 31 cases with stages I-II, and 19 cases with stages III-IV. Clinical pathological information including histopathological diagnosis and tumor-node-metastasis (TNM) stage was extracted from medical records.

\section{Immunohistochemistry staining}

Immunostaining was performed using the avidinbiotin-peroxidase complex method (SP9001; Zhongshan Goldenbridge Biotechnology, Beijing, People's Republic of China). Antigen retrieval for SPAG9 was conducted by heating the slides to $121^{\circ} \mathrm{C}$ for 90 seconds in a citrate buffer ( $\mathrm{pH}$ 6.0) and, for ELK1, was conducted by heating the slides to $100^{\circ} \mathrm{C}$ for 20 minutes in ethylene diamine tetracetic acid ( $\mathrm{pH} 9.0$ ) buffer. Next, endogenous hydrogen peroxidase was blocked by immersion in 3\% hydrogen peroxide for 10 minutes. Goat serum was used to block nonspecific binding $37^{\circ} \mathrm{C}$ for 15 minutes. Then tissue sections were incubated with SPAG9 antibody (1:1,000 dilution, ab12331; Abcam, Cambridge, UK) and ELK1 antibody (1:50 dilution, ab32106; Abcam) at $4^{\circ} \mathrm{C}$ overnight. The next day, the sections were washed in phosphate-buffered saline and incubated with biotinylated anti-rabbit secondary antibody and horseradish peroxidaselabeled avidin chain enzyme working solution for 15 minutes at room temperature. After washing, the peroxidase reaction was developed with 3, 3'-diaminobenzidine tetrahydrochloride according to the manufacturer's instructions. Five randomly selected views were examined per slide and 100 cells were observed per view at $400 \times$ magnification. Expression score was determined by staining intensity and immunoreactive cell percentage. SPAG9 cytoplasma staining and ELK1 nuclear staining or cytoplasma staining without staining intensity were rated as 0 , with weak staining in $\leq 25 \%$ as 1 , with moderate or strong staining in $25 \%-50 \%$ as 2 , strong staining in $50 \%-75 \%$ as 3 , and strong staining in $75 \%-100 \%$ as 4 . Tumor specimens were obtained by multiplying each score $<4$ into negative or low expression and $\geq 4$ into positive or highly expressed.

\section{Cell culture}

Human HuH7 and HepG2 cell lines were obtained from American Type Culture Collection (ATCC, Manassas, VA, USA). The human HCC cell line HCCLM3 was provided by the Liver Cancer Institute, Fudan University (Shanghai, People's Republic of China). The cells were maintained in Dulbecco's Modified Eagle's Medium (SH30243.01B; Hyclone, Logan City, UT, USA) with 10\% fetal bovine serum (10099-141-FBS; Life Technologies, Carlsbad, CA, USA), $100 \mathrm{U} / \mathrm{mL}$ penicillin, and $100 \mathrm{U} / \mathrm{mL}$ streptomycin (15140-122; Life Technologies) at $37^{\circ} \mathrm{C}$ in a humidified atmosphere containing $5 \% \mathrm{CO}_{2}$. Cells were grown on sterilized culture dishes and were passed every 3 days with $0.25 \%$ trypsin (Hyclone, SH30042.01, MD, USA).

\section{Transfection and small interfering RNA treatment}

SPAG9-specific siRNA targeting 5'-GCAATGACT CAGATGCATA-3', ELK1-specific siRNA targeting 5'-CACATCCCTTCTATCAGCGTGGATG-3', and control scrambled siRNA were chemically synthesized from RIBOBIO (1451493202, S151113152330; Guangzhou, People's Republic of China). ELK1 plasmid was purchased from Shanghai Genechem Co. (POSE144045095; Genechem, 
Shanghai, People's Republic of China). Human HCC cells were seeded in six-well plates at a concentration of $3 \times 10^{5}$ per well and grown overnight, and then the cells were transfected with SPAG9 or ELK1 siRNA at a final concentration of $50 \mathrm{nM}$ or $4 \mu \mathrm{g}$ of $E L K 1$ plasmids using lipofectamine 2000 reagent (11668-019; Invitrogen, Carlsbad, CA, USA) according to the manufacturer's instructions. The SPAG9-specific siRNA sequence was 5'-GCAAUGACUCAGAUGCAUA-3' and 5'-UAUGCAUCAUCUGAGUCAUUGC-3'. The ELK1-specific siRNA sequence was 5'-CACAUCCCUU CUAUCAGCGUGGAUG-3' and 5'-CAUCCACGCUGA UAGAAGGGAUGUG-3'.

\section{Wound-healing assay}

Cells transfected with $50 \mathrm{nM} S P A G 9$ siRNA or control siRNA were seeded at a density of $1 \times 10^{6}$ per well into sixwell plates. At $95 \%$ confluency, an artificial wound was created using $10 \mu \mathrm{L}$ pipette tip. After wounding, the medium was changed to fresh serum-free medium to remove cellular debris. Photomicrographs were taken at 0, 24, and 48 hours. The distances cells migrated into the wounded area were calculated by subtracting the distance 24 hours after wound healing from the initial distance.

\section{Transwell migration and invasion assay}

The transwell assay was performed as previously described. ${ }^{10,12}$ For migration assay, $\mathrm{HuH} 7$ and HCCLM3 cells transfected with $S P A G 9$ siRNA or control siRNA were plated at $1 \times 10^{5}$ cells/well in serum-free Dulbecco's Modified Eagle's Medium in the upper chamber of a 24-well Boyden chamber with a pore size of $8 \mu \mathrm{m}$ polycarbonate membrane filters (3422; Costar, Cambridge, UK). The invasion assay was performed in the same manner as the migration assay, except that the filter was precoated with $50 \mu \mathrm{L}$ matrigel (1:5 dilution, 356234; BD Bioscience, San Jose, CA, USA). Medium containing $10 \%$ fetal bovine serum in the lower chamber served as the chemoattractant. After incubation, the cells were fixed with methanol and stained with $0.05 \%$ Giemsa. Those on the upper surface of the filter were removed with a cotton swab. The filters were then rinsed in distilled water until no additional stain leached. The cells were then air dried for 20 minutes. The number of cells was counted within a field at $200 \times$ under a light microscope. For each membrane, a total of four fields were selected at random and the numbers were averaged.

\section{Quantitative reverse transcription- polymerase chain reaction}

Total RNA was extracted from HCC cells with TRIzol ${ }^{\circledR}$ (15596026; Thermo Fisher Scientific, Waltham, MA, USA) and reverse transcribed using the first-strand cDNA synthesis kit (RT0212-03; Biomiga, San Diego, USA) according to the manufacturer's protocol. Quantitative reverse transcriptionpolymerase chain reaction (RT-PCR) was performed with the Light-cycler 480 PCR apparatus (Hoffman-La Roche Ltd, Basel, Switzerland) using SYBR Green PCR master mix (DRR014A; Takara Biotechnology, Dalian, People's Republic of China). PCR reactions were performed under the following conditions: $95^{\circ} \mathrm{C}$ for 30 seconds, 40 cycles of $95^{\circ} \mathrm{C}$ for 5 seconds, and $60^{\circ} \mathrm{C}$ for 30 seconds. The relative levels of gene expression were calculated by the $-\Delta \Delta \mathrm{Ct}$ method using $\beta$-actin as a control and expressed as $2^{-\Delta \Delta \mathrm{Ct}}$. PCR primers were used as follows: SPAG9: forward, 5'-TCCACTCATCTGTAGCCCAGT-3' and reverse, 5'-CCCATTGTCCATCAACTCCT-3'; ELK1: forward, 5'-AACTTTCAGGAGACCCGTCC-3' and reverse, 5'-CCATCCCAAGGGGTACTGTTA-3'; $\beta$-actin: forward, 5'-ACCCACACTGTGCCCATCTAC-3' and reverse, 5'-TCGGTGAGGATCTCATGAGGTA-3'.

\section{Western blot analysis}

Total proteins from cell lines were extracted in lysis buffer (P0013B; Beyotime, Jiangsu, People's Republic of China) with inhibitors of proteases and phosphatases (P1260; Solarbio, Beijing, People's Republic of China) and quantified using the Bradford method. Thirty five micrograms of protein were separated by sodium dodecyl sulfate polyacrylamide gel electrophoresis (10\%) (P1200; Solarbio). After transferring, the polyvinylidene fluoride membranes (IPVH00010; Millipore, Billerica, MA, USA) were incubated overnight at $4{ }^{\circ} \mathrm{C}$ with the following antibodies: SPAG9 (1:3,000, ab12331, Abcam), ELK1 (1:500, ab32106, Abcam), p-p38 (1:1,000, ab4822, Abcam), and $\beta$-actin (1:5,000, ab198991, Abcam). After five washes with Tris-buffered saline with Tween, blots were probed with secondary antibodies conjugated with horseradish peroxidase (1:5,000, ab97051, Abcam) and were visualized using enhanced chemiluminescence (ECL)plus (32132; Thermo Fisher Scientific). Finally, blots were exposed to a radiographic film.

\section{Statistical analysis}

All data were expressed as mean \pm standard deviation, and SPSS 17.0 software was used for statistical analyses. One-way analysis of variance and Student's $t$-test or Mann-Whitney test were used to analyze the significance between groups. The correlation between SPAG9 expression and ELK1 expression in HCC tissues was analyzed using Spearman rank test. A $P$-value less than 0.05 was considered statistically significant. 


\section{Results}

\section{Expression of SPAG9 is elevated in human $\mathrm{HCC}$ and associated with ELK}

The expressions of SPAG9 and ELK1 were evaluated in 50 pairs of $\mathrm{HCC}$ and corresponding noncancerous liver specimens using immunohistochemical staining. As shown in Figure 1A, SPAG9 was localized mainly within the cytoplasmic compartment, while ELK1 was distributed throughout the whole cell, and was especially in the nucleus. Higher expression of SPAG9 and ELK1 was detected in 30/50 (60\%) and 21/50 (42\%) HCC tissues compared to the corresponding noncancerous tissues, respectively.
Next, we investigated the mRNA and protein expression of $S P A G 9$ and ELK1 in HCC cell lines using Western blot and qRT-PCR. Our results indicated that $S P A G 9$ and ELK1 mRNA and protein levels were higher in both HCCLM3 and $\mathrm{HuH} 7$ cells than HepG2 cells, a non-metastatic cell line (Figure 1B and C).

Immunohistochemical staining scores indicated that the increased expressions of SPAG9 and ELK1 observed in HCC were statistically significant compared to noncancerous tissues $(P<0.001$, Figure 1D). Spearman rank analysis confirmed that ELK1 expression was positively associated with SPAG9 expression in HCC tissues (Figure 1E). As shown in Table 1,
A
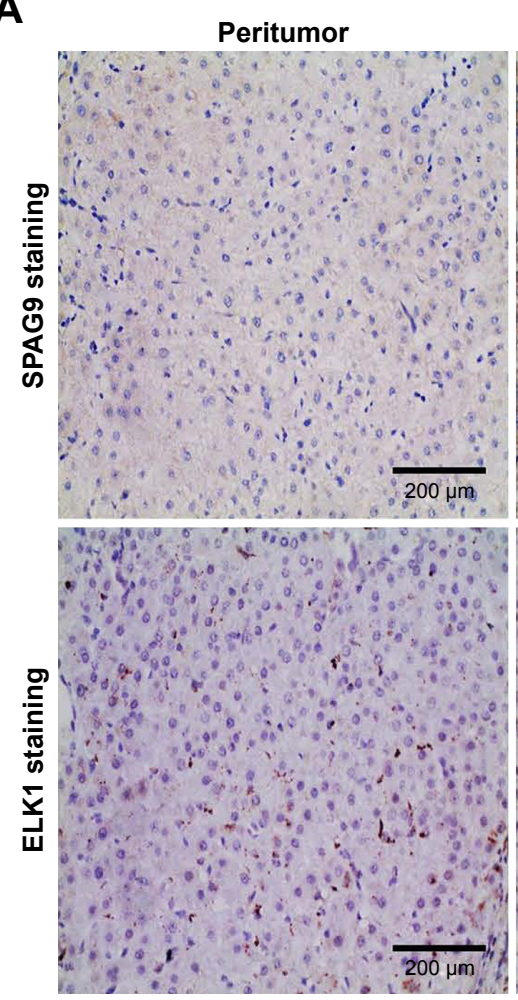

D

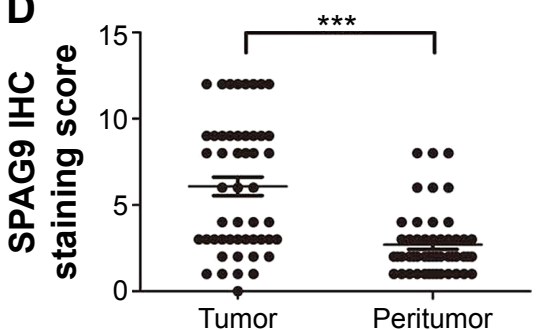

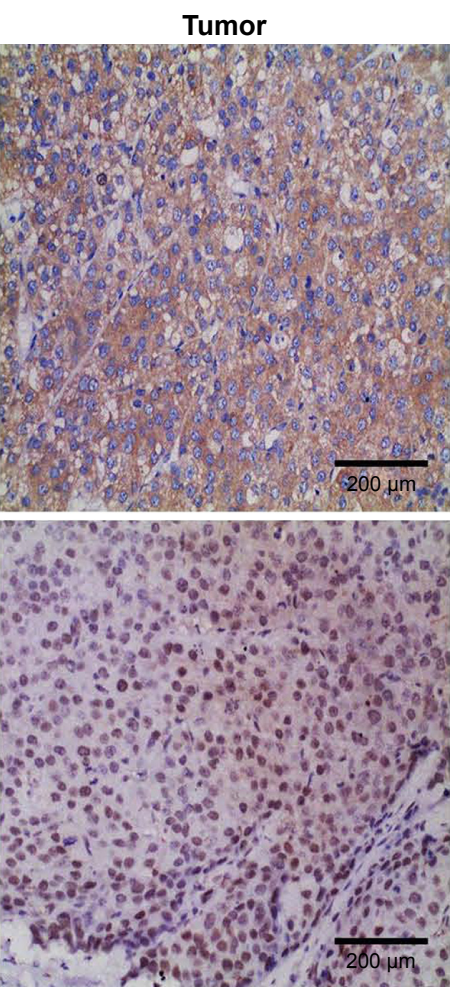

B
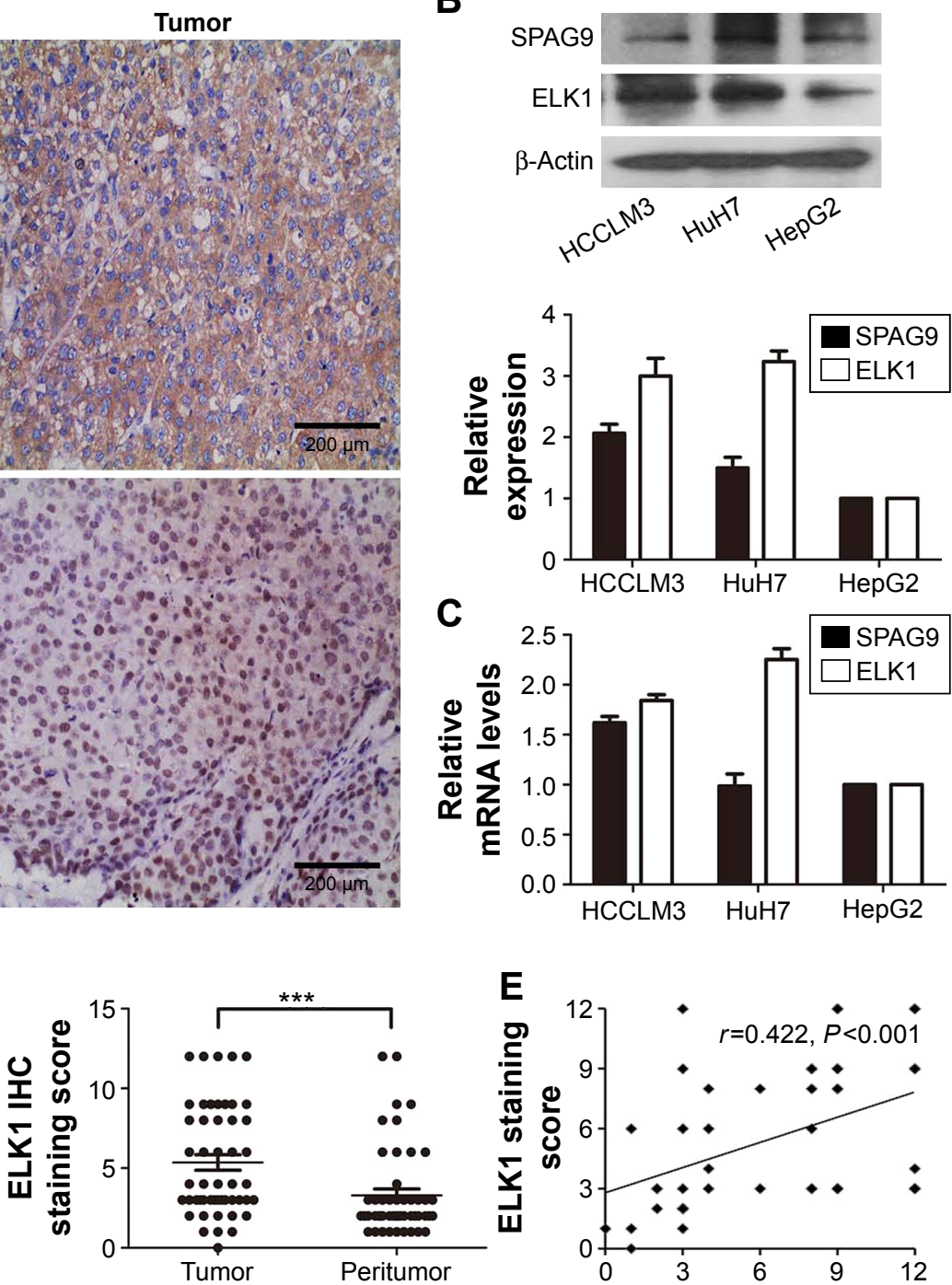

SPAG9 staining score

Figure I SPAG9 and ELKI expression in HCC tissue specimens and cell lines.

Notes: (A) IHC staining of SPAG9 and ELKI in hepatocarcinomas and adjacent noncancerous liver tissues. Weak SPAG9 and ELKI staining can be observed in the adjacent noncancerous liver tissues (left panel) compared to the strong SPAG9 cytoplasmic staining and ELKI nuclear staining in cancerous tissues (right panel) (magnification: 200 $\times$ ). (B) Protein and (C) mRNA levels of SPAG9 and ELKI were examined in HCCLM3, HuH7, and HepG2 cell lines using Western blot and qRT-PCR analyses, respectively. (D) Mann-Whitney $U$-testing of IHC scores (***P<0.00I) (the bars show standard error), and (E) Spearman rank correlation analysis of SPAG9 and ELKI expression in HCC tissues.

Abbreviations: SPAG9, sperm-associated antigen 9; IHC, immunohistochemistry; qRT-PCR, quantitative real-time polymerase chain reaction; ELKI, ETS-like gene I, tyrosine kinase; HCC, hepatocellular carcinoma. 
Table I The relationship between SPAG9 expression and the clinical features of HCC

\begin{tabular}{|c|c|c|c|c|}
\hline \multirow[t]{2}{*}{ Parameters } & \multirow[t]{2}{*}{ Total } & \multicolumn{2}{|c|}{ SPAG9 expression } & \multirow[t]{2}{*}{$P$-value } \\
\hline & & $\begin{array}{l}\text { Positive } \\
\text { n (\%) }\end{array}$ & $\begin{array}{l}\text { Negative } \\
\text { n (\%) }\end{array}$ & \\
\hline \multicolumn{5}{|l|}{ Age (year) } \\
\hline$\leq 50$ & 20 & $13(65.0)$ & $7(35.0)$ & 0.769 \\
\hline$>50$ & 30 & $17(56.7)$ & $13(43.3)$ & \\
\hline \multicolumn{5}{|l|}{ Sex } \\
\hline Male & 39 & $24(6 \mid .5)$ & $15(38.5)$ & 0.736 \\
\hline Female & 11 & $6(54.5)$ & $5(45.5)$ & \\
\hline \multicolumn{5}{|l|}{$\mathrm{HBsAg}$} \\
\hline Presence & 36 & $22(6 \mathrm{I} . \mathrm{I})$ & 14 (38.9) & 0.283 \\
\hline Absence & 14 & $8(57.1)$ & $6(42.9)$ & \\
\hline \multicolumn{5}{|l|}{ Cirrhosis } \\
\hline Presence & 30 & $21(70.0)$ & $9(30.0)$ & 0.089 \\
\hline Absence & 20 & $9(45.0)$ & II (55.0) & \\
\hline \multicolumn{5}{|l|}{ TNM stage } \\
\hline $1+I I$ & 31 & I5 (48.4) & $16(51.6)$ & $0.032^{*}$ \\
\hline III+IV & 19 & 15 (78.9) & $4(21.1)$ & \\
\hline \multicolumn{5}{|c|}{ Relapse or metastasis } \\
\hline Presence & 30 & $22(73.3)$ & $8(26.7)$ & $0.018^{*}$ \\
\hline Absence & 20 & $8(40.0)$ & $12(60.0)$ & \\
\hline \multicolumn{5}{|l|}{ ELKI status } \\
\hline Positive & 21 & I8 (85.7) & $3(14.3)$ & $0.002 *$ \\
\hline Negative & 29 & $12(4 \mid .4)$ & $17(58.6)$ & \\
\hline
\end{tabular}

SPAG9 overexpression was positively correlated with TNM staging, metastasis, and relapse parameters of HCC patients $\left(\chi^{2}=4.585, P=0.032 ; \chi^{2}=5.556, P=0.018\right)$. These results suggest that SPAG9 expression is positively associated with ELK1 and highly correlated to HCC metastasis.

\section{Knockdown of SPAG9 expression suppresses HCC cell migration and invasion}

To further explore the role of $S P A G 9$ in HCC metastasis, the migration and invasion activity of HCCLM3 and $\mathrm{HuH} 7$ cells was analyzed using a wound-healing and transwell assays, respectively. Interference of $S P A G 9$ expression was achieved by transfecting cells with $S P A G 9$-specific siRNA (Figure 2A and B). The results from the wound-healing assay showed that the migratory potential of $S P A G 9$-silenced HCCLM3 and HuH7 cells was significantly reduced compared to scramble RNA-transfected cells (Figure 2C). The results from the transwell migration assay showed that SPAG9 siRNA-transfected cells had a significant reduction in migration compared to HCCLM3 and HuH7 control cells ( $116 \pm 15$ vs $80 \pm 9 ; 326 \pm 30$ vs $141 \pm 20$ cells per fields, $P<0.05$ ) (Figure 2D). Matrigel invasion assays also showed that
SPAG9 knockdown inhibited cell invasion in HCCLM3 and $\mathrm{HuH} 7$ cells compared to the controls ( $49 \pm 9$ vs $88 \pm 12 ; 81 \pm 10$ vs $205 \pm 15, P<0.05$ ) (Figure 2D). These results demonstrate that $S P A G 9$ is involved in the migration and invasion of HCCLM3 and HuH7 cells.

\section{SPAG9 knockdown decreases ELKI expression and inhibits $\mathrm{p} 38$ activation}

Given that ELK1 expression was positively associated with SPAG9 expression in HCC tissues and cell lines, we investigated whether SPAG9 was able to modulate ELK1 expression. As depicted in Figure 3A and B, siRNA-mediated silencing of $S P A G 9$ significantly decreased $E L K 1$ expression at both the protein and mRNA level compared to cells transfected with scrambled siRNA. Furthermore, we examined the effect of SPAG9 silencing on p38 activation in HCC cells. We observed a strong reduction in phosphorylated $\mathrm{p} 38$ following SPAG9 knockdown (Figure 3C). These results suggest that $S P A G 9$ may modulate ELK1 expression and p38 activation.

\section{SPAG9-induced migration and invasion of HCC cells are dependent on ELKI expression and ELKI knockdown suppresses HCC cell migration and invasion}

To further assess the functional relationship between ELKI and $S P A G 9$, we transfected SPAG9-silenced $\mathrm{HuH} 7$ cells with an $E L K 1$ plasmid to upregulate $E L K 1$ expression in these $\mathrm{HuH} 7$ cells. We observed that ELK1 overexpression attenuated the inhibitory effects of $S P A G 9$ siRNA on cell migration and invasion $(P<0.05)$ (Figure 4A and 4B). Furthermore, the migration and invasion activity of $\mathrm{HuH} 7$ cells was inhibited by transfection with $E L K 1$ siRNA $(P<0.05)$ (Figure $4 C$ ). As shown in Figure 4D, the protein level of ELK1 was significantly decreased 48 hours after siRNA transfection and the SPAG9 expression was not changed. These results suggest that $S P A G 9$-induced migration and invasion of HCC cells are dependent on ELK1 expression, and ELK1 knockdown suppresses HCC cells migration and invasion.

\section{Discussion}

Given that metastasis plays an important role in recurrence and poor prognosis of $\mathrm{HCC}$, it is necessary to identify novel therapeutic strategies for treatment of metastasis. Initially, it was reported that SPAG9 was involved in sperm-egg interactions. ${ }^{22}$ Recently, in vitro and in vivo studies have shown that siRNA-mediated knockdown of $S P A G 9$ reduces tumor growth, as well as invasion and migration of various 

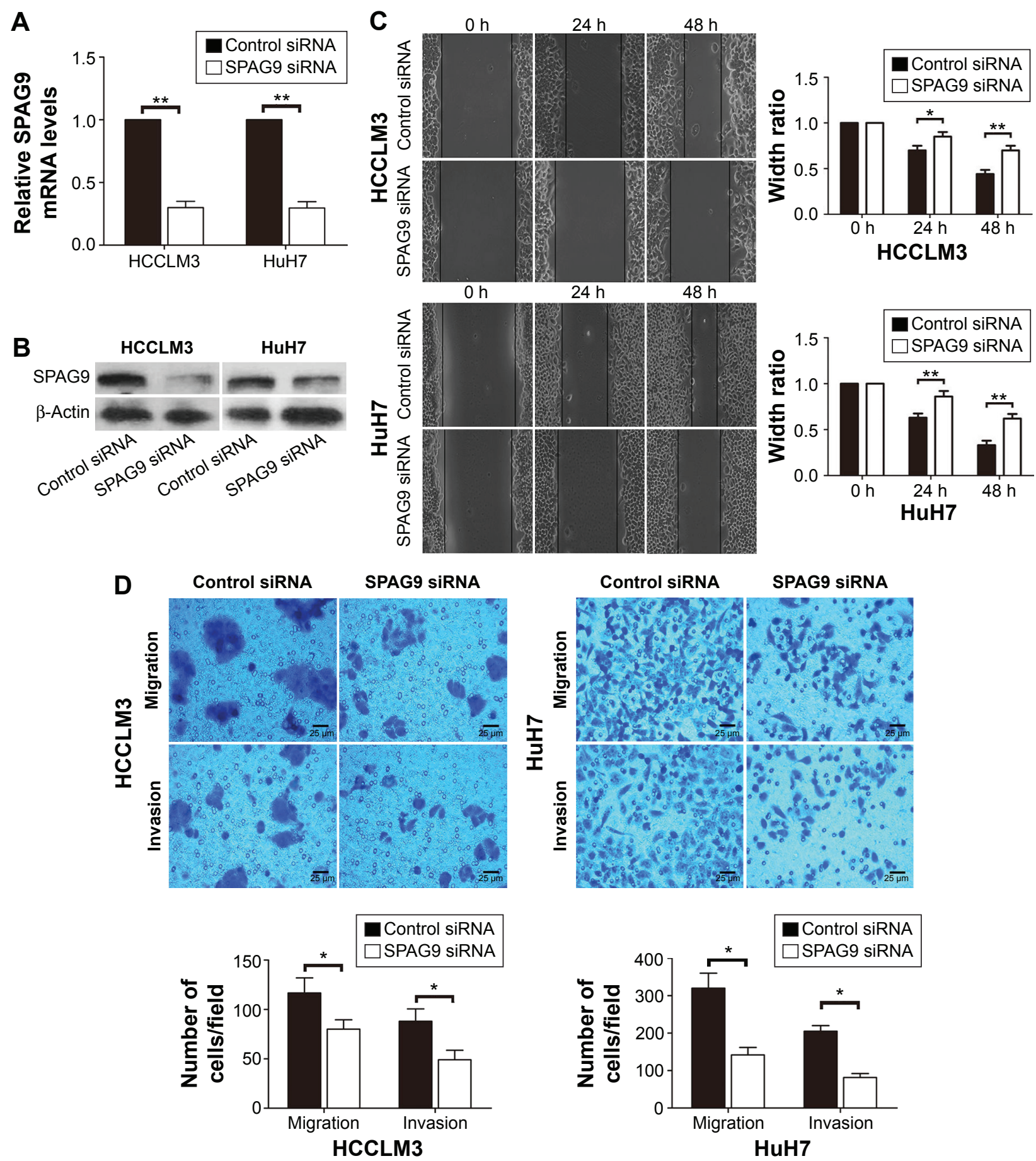

Figure 2 Reduction in SPAG9 expression suppresses cell migration and invasion of HCC cell lines.

Notes: (A and B) SPAG9 siRNA-treated cells had decreased mRNA (A) and protein (B) levels compared to the control cells. (C) Results from the wound-healing assay showed that SPAG9 depletion decreased cell migration of HCCLM3 and HuH7 cells. (D) Results from the transwell migration and matrigel invasion assay showed that SPAG9 depletion decreased cell migration and invasion of $\mathrm{HCCLM} 3$ and $\mathrm{HuH7}$ cells; $* P<0.05, * * P<0.01$.

Abbreviations: SPAG9, sperm-associated antigen 9; siRNA, small interfering RNA; HCC, hepatocellular carcinoma; h, hour.

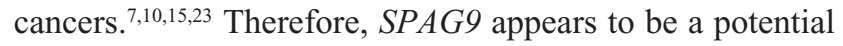
therapeutic target for HCC metastasis.

In this study, we found that SPAG9 was overexpressed in $60 \%(30 / 50)$ of human HCC tissues and was correlated with TNM staging. Moreover, SPAG9 expression was associated with relapse and metastasis in HCC patients (Table 1).
Interference of $S P A G 9$ reduced migration and invasion of HCC cell lines (HCCLM3 and HuH7) indicating that $S P A G 9$ is involved in the metastasis of HCC. Metastasis is a multistep process, including epithelial mesenchymal transition, mesenchymal epithelial transition, migration, and invasion. ${ }^{24}$ Matrix metalloproteinase-9 (MMP9) was 

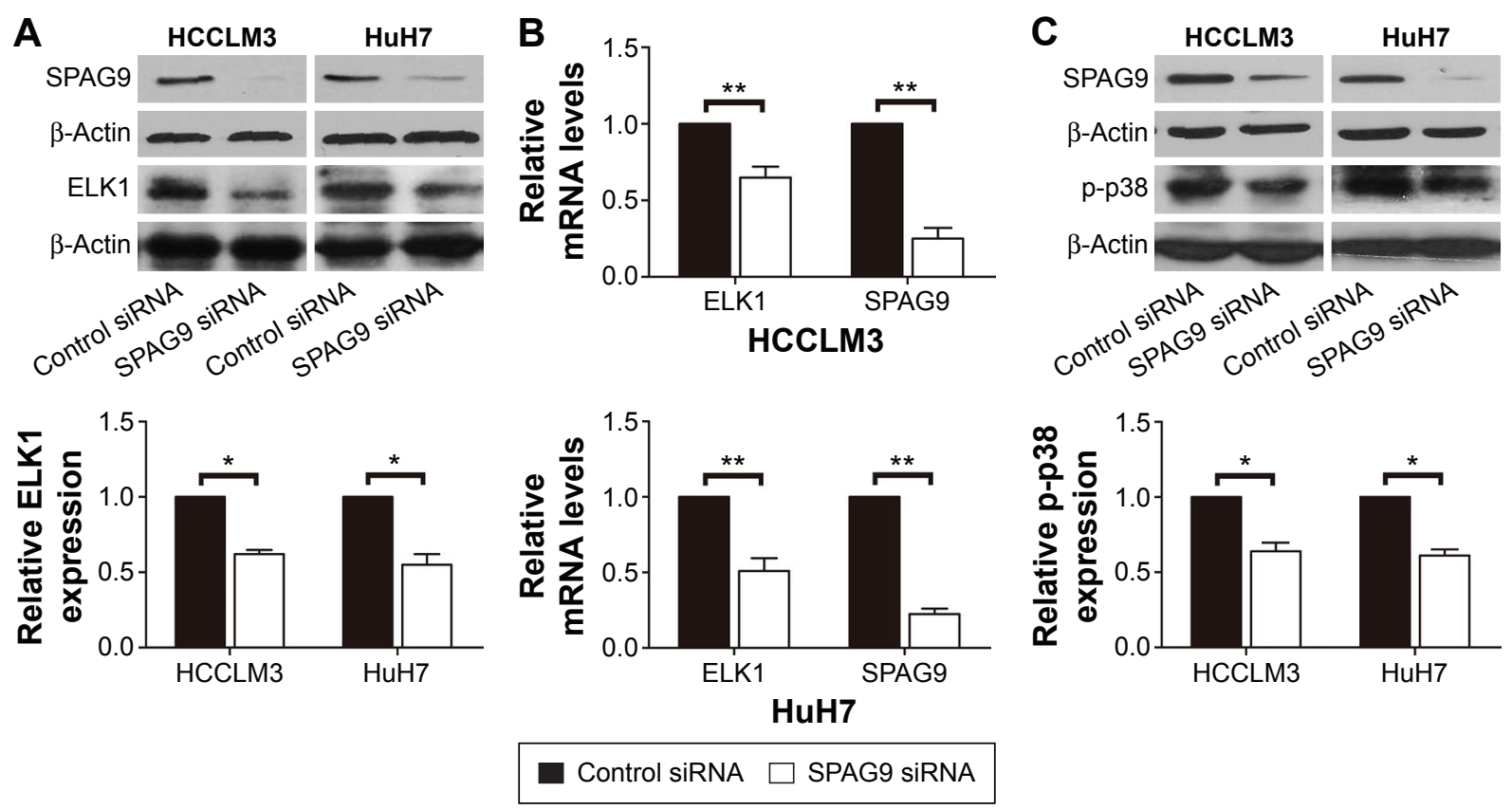

Figure 3 SPAG9 regulates ELKI expression and $\mathrm{p} 38$ activation in $\mathrm{HCC}$ cell lines.

Notes: (A) Western blot and (B) qRT-PCR analyses revealed that siRNA knockdown of SPAG9 decreased protein and mRNA levels of SPAG9 and ELKI, respectively, in HCCLM3 and HuH7 cell lines. (C) Western blot analysis showed that SPAG9 depletion decreased SPAG9 and p-p38 levels in HCCLM3 and HuH7 cells.

Abbreviations: SPAG9, sperm-associated antigen 9; siRNA, small interfering RNA; qRT-PCR, quantitative real-time polymerase chain reaction; ELKI, ETS-like gene I, tyrosine kinase; p-p38, phosphorylated protein-38; HCC, hepatocellular carcinoma.
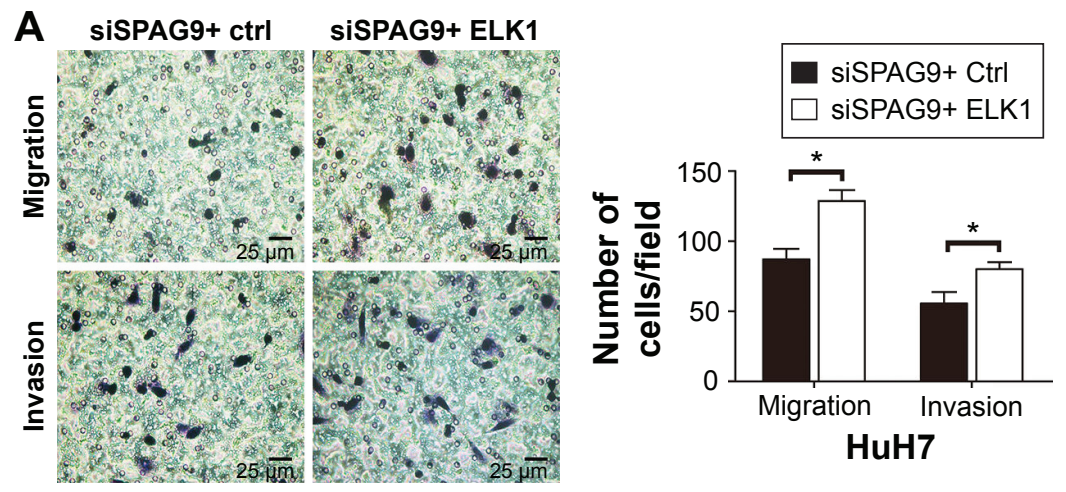

B
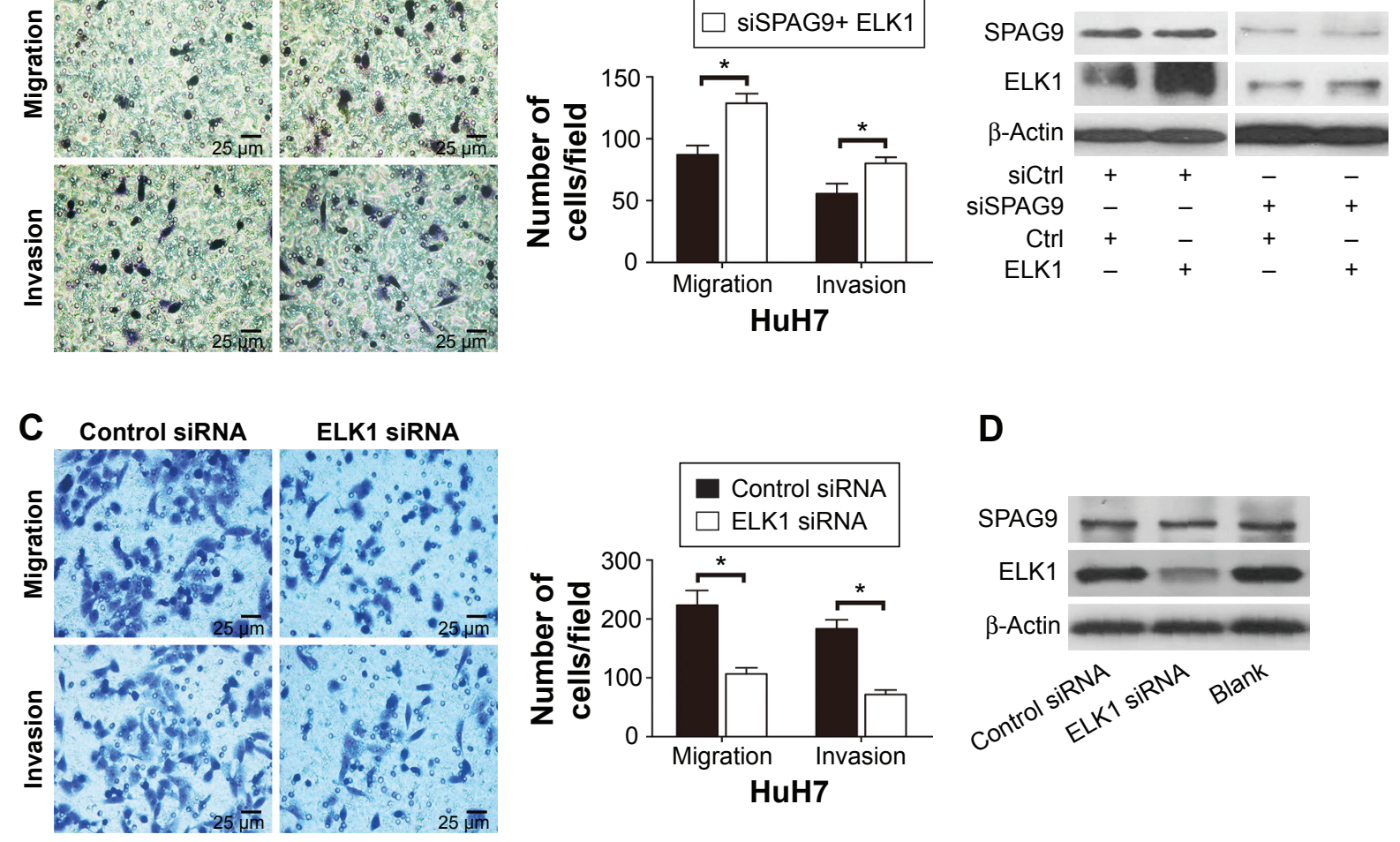

D

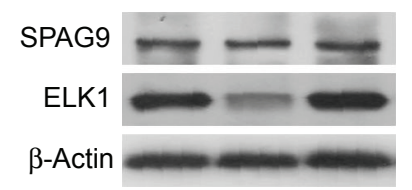

Control ELRA SiRn Blank

\section{$\mathrm{HuH7}$}

Figure 4 SPAG9-induced migration and invasion of HCC cells is dependent on ELKI.

Notes: (A) Transwell migration and matrigel invasion assay revealed that ELKI transfection rescued the inhibition in cell migration and invasion induced by SPAG9 depletion. (B) The expression of ELKI was significantly upregulated in the siCtrl + ELK group and siSPAG9+ ELKI group compared to the controls. (C) Transwell migration and matrigel invasion assay revealed that ELKI depletion decreased the migration and invasion in HuH7 cells. (D) Western blot showed that siRNA knockdown of ELKI decreased the protein level of ELKI, while SPAG9 expression was not changed in HuH7 cells. *P<0.05.

Abbreviations: SPAG9, sperm-associated antigen 9; siSPAG9, SPAG9 siRNA; siCtrl, control siRNA; siRNA, small interfering RNA; ELKI, ETS-like gene I, tyrosine kinase; HCC, hepatocellular carcinoma. 
reported to be downregulated after $S P A G 9$ knockdown, indicating that $S P A G 9$ may regulate invasion through MMP9 in astrocytomas and lung cancers. ${ }^{9,11}$ Furthermore, studies have reported that MMP2, MMP9, and vascular endothelial growth factor molecules participate in SPAG9-mediated migration and invasion of prostate cancer cells. ${ }^{12}$ However, the molecular pathways involved in SPAG9-mediated metastasis in HCC remain unknown.

The ETS family proteins affect the expression of several oncogenes and tumor suppressor genes directly by regulating their promoters or indirectly through protein-protein interactions. ${ }^{25}$ One such member, ELK1, has been reported to control breast cancer migration by regulating a set of target genes "unique" to $E L K 1 .{ }^{18}$ In addition, ELK1 activation is involved in bombesin-induced prostate cancer cell growth. ${ }^{26}$ Loss of ELK1 expression inhibited the expression of protein kinase $\mathrm{C}$ alpha and decreased the cell migration and invasion ability of $\mathrm{HCC}^{20}$ In this study, we found ELK1 was overexpressed in $42 \%(21 / 50)$ of HCC tissues specimens. In addition, $S P A G 9$ knockdown reduced ELK1 expression, while $E L K 1$ overexpression reversed the inhibitory effects of SPAG9 knockdown on cell migration and invasion, and ELK1 depletion hindered the metastasis ability of $\mathrm{HuH} 7$ cells. This result indicated that SPAG9-enhanced migration and invasion may depend on ELK1 expression. Experimental evidence demonstrated that the p38 MAPK pathway is involved in metastasis of tumor cells. ${ }^{27}$ SPAG9 acts as a scaffolding protein, which can activate $\mathrm{p} 38$ signaling. ${ }^{28,29}$ It promotes the invasion of gastric and prostate cancer through the upregulation of $\mathrm{p} 38 .{ }^{15,30}$ In this study, we observed a reduction in $\mathrm{p} 38$ after $S P A G 9$ interference. Given that $E L K 1$ was one of the target genes of p38 signaling, ${ }^{17,31}$ we speculated that SPAG9induced migration and invasion may be partly modulated by p38/ELK1 signaling in HCC.

\section{Conclusion}

Our study showed that SPAG9 and ELK1 were upregulated in HCC tissue samples and that SPAG9 overexpression was positively correlated with HCC metastasis. Furthermore, $S P A G 9$-induced migration and invasion were dependent on $E L K 1$ expression, suggesting that $S P A G 9$ may be a potential anti-metastasis target for HCC therapy.

\section{Acknowledgments}

This work was supported by the National Science Foundation of Zhejiang Province (LY14H200001), Medicines Health Platform Key Project of Zhejiang Province (2013ZDA024), and Medicines Health Platform Plan Project of Zhejiang Province (2015DTA018).

\section{Disclosure}

The authors report no conflicts of interest in this work.

\section{References}

1. Forner A, Llovet JM, Bruix J. Hepatocellular carcinoma. Lancet. 2012; 379(9822):1245-1255.

2. Poon RT, Ng IO, Fan ST, et al. Clinicopathologic features of longterm survivors and disease-free survivors after resection of hepatocellular carcinoma: a study of a prospective cohort. J Clin Oncol. 2001; 19(12):3037-3044.

3. Simpson AJ, Caballero OL, Jungbluth A, Chen YT, Old LJ. Cancer/ testis antigens, gametogenesis and cancer. Nat Rev Cancer. 2005;5(8): 615-625.

4. Fratta E, Coral S, Covre A, et al. The biology of cancer testis antigens: putative function, regulation and therapeutic potential. Mol Oncol. 2011;5(2):164-182.

5. Suri A, Saini S, Sinha A, et al. Cancer testis antigens: a new paradigm for cancer therapy. Oncoimmunology. 2012;1(7):1194-1196.

6. Kanojia D, Garg M, Gupta S, Gupta A, Suri A. Sperm-associated antigen 9, a novel biomarker for early detection of breast cancer. Cancer Epidemiol Biomarkers Prev. 2009;18(2):630-639.

7. Kanojia D, Garg M, Gupta S, Gupta A, Suri A. Sperm-associated antigen 9 is a novel biomarker for colorectal cancer and is involved in tumor growth and tumorigenicity. Am J Pathol. 2011;178(3):1009-1020.

8. Garg M, Kanojia D, Suri S, Suri A. Small interfering RNA-mediated down-regulation of SPAG9 inhibits cervical tumor growth. Cancer. 2009; 115(24):5688-5699.

9. Yi F, Ni W, Liu W, et al. SPAG9 is overexpressed in human astrocytoma and promotes cell proliferation and invasion. Tumour Biol. 2013; 34(5):2849-2855.

10. Sinha A, Agarwal S, Parashar D, et al. Down regulation of SPAG9 reduces growth and invasive potential of triple-negative breast cancer cells: possible implications in targeted therapy. J Exp Clin Cancer Res. 2013;32:69.

11. Wang Y, Dong Q, Miao Y, Fu L, Lin X, Wang E. Clinical significance and biological roles of SPAG9 overexpression in non-small cell lung cancer. Lung Cancer. 2013;81(2):266-272.

12. Chen F, Lu Z, Deng J, et al. SPAG9 expression is increased in human prostate cancer and promotes cell motility, invasion and angiogenesis in vitro. Oncol Rep. 2014;32(6):2533-2540.

13. Jagadish N, Rana R, Selvi R, et al. Characterization of a novel human sperm-associated antigen 9 (SPAG9) having structural homology with c-Jun N-terminal kinase Interacting protein. Biochem J. 2005;389(Pt 1): $73-82$.

14. Engstrom W, Ward A, Moorwood K. The role of scaffold proteins in JNK signalling. Cell Prolif. 2010;43(1):56-66.

15. Jilg CA, Ketscher A, Metzger E, et al. PRK1/PKN1 controls migration and metastasis of androgen-independent prostate cancer cells. Oncotarget. 2014;5(24):12646-12664.

16. Cuenda A, Rousseau S. p38 MAP-kinases pathway regulation, function and role in human diseases. Biochim Biophys Acta. 2007;1773(8): 1358-1375.

17. Ferreiro I, Barragan M, Gubern A, Ballestar E, Joaquin M, Posas F. The p38 SAPK is recruited to chromatin via its interaction with transcription factors. J Biol Chem. 2010;285(41):31819-31828.

18. Odrowaz Z, Sharrocks AD. The ETS transcription factors ELK1 and GABPA regulate different gene networks to control MCF10A breast epithelial cell migration. PloS One. 2012;7(12):e49892.

19. Odrowaz Z, Sharrocks AD. ELK1 uses different DNA binding modes to regulate functionally distinct classes of target genes. PLoS Genet. 2012;8(5):e1002694. 
20. Hsieh YH, Wu TT, Tsai JH, Huang CY, Hsieh YS, Liu JY. PKCalpha expression regulated by Elk-1 and MZF-1 in human HCC cells. Biochem Biophys Res Commun. 2006;339(1):217-225.

21. Behrens P, Rothe M, Florin A, Wellmann A, Wernert N. Invasive properties of serous human epithelial ovarian tumors are related to Ets-1, MMP-1 and MMP-9 expression. Int J Mol Med. 2001;8(2):149-154.

22. Jagadish N, Rana R, Mishra D, Kumar M, Suri A. Sperm associated antigen 9 (SPAG9): a new member of c-Jun NH2-terminal kinase (JNK) interacting protein exclusively expressed in testis. Keio J Med. 2005;54(2):66-71.

23. Garg M, Kanojia D, Khosla A, et al. Sperm-associated antigen 9 is associated with tumor growth, migration, and invasion in renal cell carcinoma. Cancer Res. 2008;68(20):8240-8248.

24. Hynes RO. Metastatic potential: generic predisposition of the primary tumor or rare, metastatic variants or both? Cell. 2003;113(7):821-823.

25. Oikawa T. ETS transcription factors: possible targets for cancer therapy. Cancer Sci. 2004;95(8):626-633.

26. Xiao D, Qu X, Weber HC. GRP receptor-mediated immediate early gene expression and transcription factor Elk-1 activation in prostate cancer cells. Regul Pept. 2002;109(1-3):141-148.
27. del Barco Barrantes I, Nebreda AR. Roles of p38 MAPKs in invasion and metastasis. Biochem Soc Trans. 2012;40(1):79-84.

28. Kelkar N, Standen CL, Davis RJ. Role of the JIP4 scaffold protein in the regulation of mitogen-activated protein kinase signaling pathways. Mol Cell Biol. 2005;25:2733-2743.

29. Pan CQ, Sudol M, Sheetz M, Low BC. Modularity and functional plasticity of scaffold proteins as p(1)acemakers in cell signaling. Cell Signal. 2012;24:2143-2165.

30. Miao Z-F, Wang Z-N, Zhao T-T, et al. Overexpression of SPAG9 in human gastric cancer is correlated with poor prognosis. Virchows Arch. 2015;467(5):525-533.

31. Mut M, Lule S, Demir O, Kurnaz IA, Vural I. Both mitogen-activated protein kinase (MAPK)/extracellular-signal-regulated kinases (ERK) $1 / 2$ and phosphatidylinositide-3-OH kinase (PI3K)/Akt pathways regulate activation of E-twenty-six (ETS)-like transcription factor 1 (Elk-1) in U138 glioblastoma cells. Int J Biochem Cell Biol. 2012; 44(2):302-310.
OncoTargets and Therapy

\section{Publish your work in this journal}

OncoTargets and Therapy is an international, peer-reviewed, open access journal focusing on the pathological basis of all cancers, potential targets for therapy and treatment protocols employed to improve the management of cancer patients. The journal also focuses on the impact of management programs and new therapeutic agents and protocols on

\section{Dovepress}

patient perspectives such as quality of life, adherence and satisfaction. The manuscript management system is completely online and includes a very quick and fair peer-review system, which is all easy to use. Visit http://www.dovepress.com/testimonials.php to read real quotes from published authors. 